\title{
Effects of static charging and exfoliation of layered crystals
}

\author{
M. Topsakal ${ }^{1}$ and S. Ciraci ${ }^{1,2, *}$ \\ ${ }^{1}$ UNAM-Institute of Materials Science and Nanotechnology, Bilkent University, Ankara TR-06800, Turkey \\ ${ }^{2}$ Department of Physics, Bilkent University, Ankara TR-06800, Turkey
}

(Received 12 August 2011; revised manuscript received 9 January 2012; published 20 January 2012)

\begin{abstract}
Using a first-principle plane-wave method we investigate the effects of static charging on the structural, electronic, and magnetic properties of suspended, single-layer graphene, graphane, fluorographene, BN, and $\mathrm{MoS}_{2}$ in a honeycomb structure. The limitations of periodic boundary conditions in the treatment of negatively charged layers are clarified. Upon positive charging, the band gaps between the conduction and valence bands increase, but the single-layer nanostructures become metallic owing to the Fermi level dipping below the maximum of valence band. Moreover, their bond lengths increase, leading to phonon softening. As a result, the frequencies of Raman active modes are lowered. A high level of positive charging leads to structural instabilities in single-layer nanostructures, since their specific phonon modes attain imaginary frequencies. Similarly, excess positive charge is accumulated at the outermost layers of metallized $\mathrm{BN}$ and $\mathrm{MoS}_{2}$ sheets comprising a few layers. Once the charging exceeds a threshold value, the outermost layers are exfoliated. Charge relocation and repulsive force generation are in compliance with classical theories.
\end{abstract}

DOI: 10.1103/PhysRevB.85.045121

PACS number(s): 36.40.Wa, 31.15.A-, 73.20.-r, 81.05.ue

\section{INTRODUCTION}

Single-layer graphene, ${ }^{1}$ graphane $\mathrm{CH},{ }^{2,3}$ fluorographene $\mathrm{CF}^{4,5} \mathrm{BN},{ }^{6,7}$ and $\mathrm{MoS}_{2}{ }^{8,9}$ have displayed unusual chemical and physical properties for future nanotechnology applications. Furthermore, the properties of these nanomaterials can be modified by creating excess electrical charge. For example, linear crossing of bands of graphene at the Fermi level gives rise to electron-hole symmetry, whereby under bias voltage the charge carriers can be tuned continuously between electrons and holes in significant concentrations. ${ }^{10}$ This way, the conductivity of graphene can be monitored. A similar situation leading to excess electrons or holes can also be achieved through doping with foreign atoms. ${ }^{1-15}$ Layered materials can be exfoliated under excessive charging, which is created by photoexcitation for very short times. ${ }^{16,17}$ It is proposed that the femtosecond laser pulses rapidly generate hot electron gas, which spills out leaving behind a positively charged graphite slab. Eventually, charged outermost layers of graphite are exfoliated. ${ }^{17}$

Recently, the effects of charging of graphene have been treated in different studies. Ekiz et al. ${ }^{18}$ showed that oxidized graphene domains, which become insulating upon oxidation, change back to the metallic state using electrical stimulation. Theoretically, based on first-principles calculations, it has been shown that the binding energy and magnetic moments of adatoms adsorbed on graphene can be modified through static charging. ${ }^{15,19}$ The possibility of transforming the electronic structure of one species to another through gating modeled by charging has been pointed out. ${ }^{20}$ It is argued that diffusion of adsorbed oxygen atoms on graphene can be modified through charging. ${ }^{21}$ We found that pseudopotential plane-wave calculations of charged surfaces using periodically repeating layers are sensitive to the vacuum spacing between adjacent cells and have limited applicability. ${ }^{15}$

In this paper we investigate the effect of static charging on suspended (or free-standing) single-layer nanostructures, such as graphene, graphane $(\mathrm{CH})$, fluorographene (fully fluorinated graphene, $\mathrm{CF}$ ), boron nitride $(\mathrm{BN})$, and molybdenum disulfide $\left(\mathrm{MoS}_{2}\right)$. All these honeycomb nanostructures have two-dimensional (2D) hexagonal lattices. First, we examine how the size of the "vacuum" potential between layers affects the calculated properties of the negatively charged singlelayer nanostructures when treated using periodic boundary conditions. We then investigate the effect of charging on the electronic energy band structure and atomic structure. We show that the bond lengths and hence 2D lattice constants increase as a result of electron removal from the single layer. Consequently, phonons soften and the frequencies of Raman active modes are lowered. As a result of electron removal, three-layer, wide-band-gap $\mathrm{BN}$ and $\mathrm{MoS}_{2}$ sheets are metallized and excess positive charge is accumulated mainly at the outermost atomic layers. Owing to the Coulomb force, those layers start to repel each other. When the weak van der Waals (vdW) attraction is exceeded, the repulsive Coulomb force initiates the exfoliation.

\section{METHOD}

The present results are obtained by performing firstprinciples plane-wave calculations carried out within spinpolarized and spin-unpolarized density functional theory (DFT) using projector-augmented wave potentials. ${ }^{22}$ The exchange correlation potential is approximated by the generalized gradient approximation. ${ }^{23}$ For a better account of weak interlayer attraction in layered crystals, van der Waals (vdW) interaction is also taken into account. ${ }^{24} \mathrm{~A}$ plane-wave basis set with kinetic energy cutoff of $500 \mathrm{eV}$ is used. All atomic positions and lattice constants are optimized by using the conjugate gradient method, where the total energy and atomic forces are minimized. The convergence for energy is chosen as $10^{-5} \mathrm{eV}$ between two steps, and the maximum force allowed on each atom is less than $0.01 \mathrm{eV} / \AA$. The Brillouin zone $(\mathrm{BZ})$ is sampled by $(15 \times 15 \times 5)$ special $\mathbf{k}$ points for the primitive unit cell. Calculations for neutral, as well as charged systems, are carried out by using the VASP package. ${ }^{25}$

Two-dimensional single layers or slabs and a vacuum space $s$ between them are repeated periodically along the perpendicular $z$ direction. The amount of charging is specified as either positive charging [i.e., electron depletion $(Q>0)$ ], 
or negative charging [i.e., excess electrons $(Q<0)$ ], in units of \pm one electron (e) per unit cell. Average surface charge density is specified as $\bar{\sigma}=Q / A$, which is the charge per unit area, where $A$ is the area of the unit cell. Normally, periodic boundary conditions realized by repeating charged supercells have a divergent electric potential energy and have drawbacks and limitations, which have been the subject of several studies in the past. To achieve the convergence of electronic potential, additional neutralizing background charge is applied. ${ }^{26,27}$ Recently, error bars in computations due to compensating charge have been estimated. ${ }^{20}$ The dipole corrections can be carried out for cubic structures, if a finite electric dipole moment builds in the unit cell. ${ }^{28,29}$ Monopole and dipole corrections are also treated self-consistently. ${ }^{30}$ Various charged structures have been also treated by using different approaches and computational methods. ${ }^{31-37}$ Owing to those theoretical advances, studies on charged systems can now reveal useful information, when treated carefully.

\section{CHARGING OF SUSPENDED SINGLE LAYERS}

The negative and positive charging of suspended singlelayer graphene, $\mathrm{CH}, \mathrm{CF}, \mathrm{BN}$, and $\mathrm{MoS}_{2}$ are treated using supercell method. In Fig. 1(a) we describe $\mathrm{MoS}_{2}$ single layers, which are periodically repeated along the $z$ direction and separated by a vacuum spacing $s$ between the adjacent outermost sulfur planes. In Figs. 1(b) and 1(c) the self-consistent electronic potential energy, $V_{e l}(\mathbf{r})$ is averaged in the planes perpendicular to the $z$ axis to obtain planarly averaged one-dimensional (1D) potential energy $\bar{V}_{e l}(z)$ for different values of $s$.

In the vacuum region, the electronic potential energy $\bar{V}_{e l}(z)$ strongly depends on the vacuum spacing $s$. For an infinitely large single plane having excess charge $Q>0$ per cell, the potential energy in the vacuum region is linear, if it is not periodically repeating. Thus, as $z \rightarrow \infty, \bar{V}_{e l}(z \rightarrow$ $\infty) \rightarrow+\infty$ as schematically shown in Fig. 1(b). However, for a periodically repeating single layers (within the periodic boundary conditions) the potential energy is symmetric with respect to the center of vacuum spacing and it passes through a maximum at $s / 2$. The maximum value of the potential increases with increasing $s$ in Fig. 1(b).

In contrast, for a negatively ( $Q<0$ per cell) charged and infinite $\mathrm{MoS}_{2}$ single layer, a reverse situation occurs as shown in Fig. 1(c). Namely, $\bar{V}_{e l}(z \rightarrow \infty) \rightarrow-\infty$ linearly, if the $\mathrm{MoS}_{2}$ single layer is not periodically repeating. Notably, the energy of a finite-size, single-layer nanostructure (i.e., a flake) does not diverge, but has finite value for large $z$ both for the $Q>0$ and $Q<0$ cases. On the other hand, for periodically repeating single layers within the periodic boundary conditions, potential energies are symmetric with respect to the center of vacuum spacing and they pass through a minimum at $s / 2$. This way a potential well is formed in the vacuum region between two adjacent layers. Normally, the depth of this well increases with increasing negative charging and $s$. At a critical value of negative charge, the self-consistent potential energy $V(\mathbf{r})$ including electronic and exchange-correlation potential energies dip below the Fermi level [even if $\bar{V}_{e l}(z)>E_{F}$ ] and eventually electrons start to occupy the states localized in the quantum well. Such a situation is described in Figs. 1(d)-1(f). Of course, this situation is an artifact of the method using
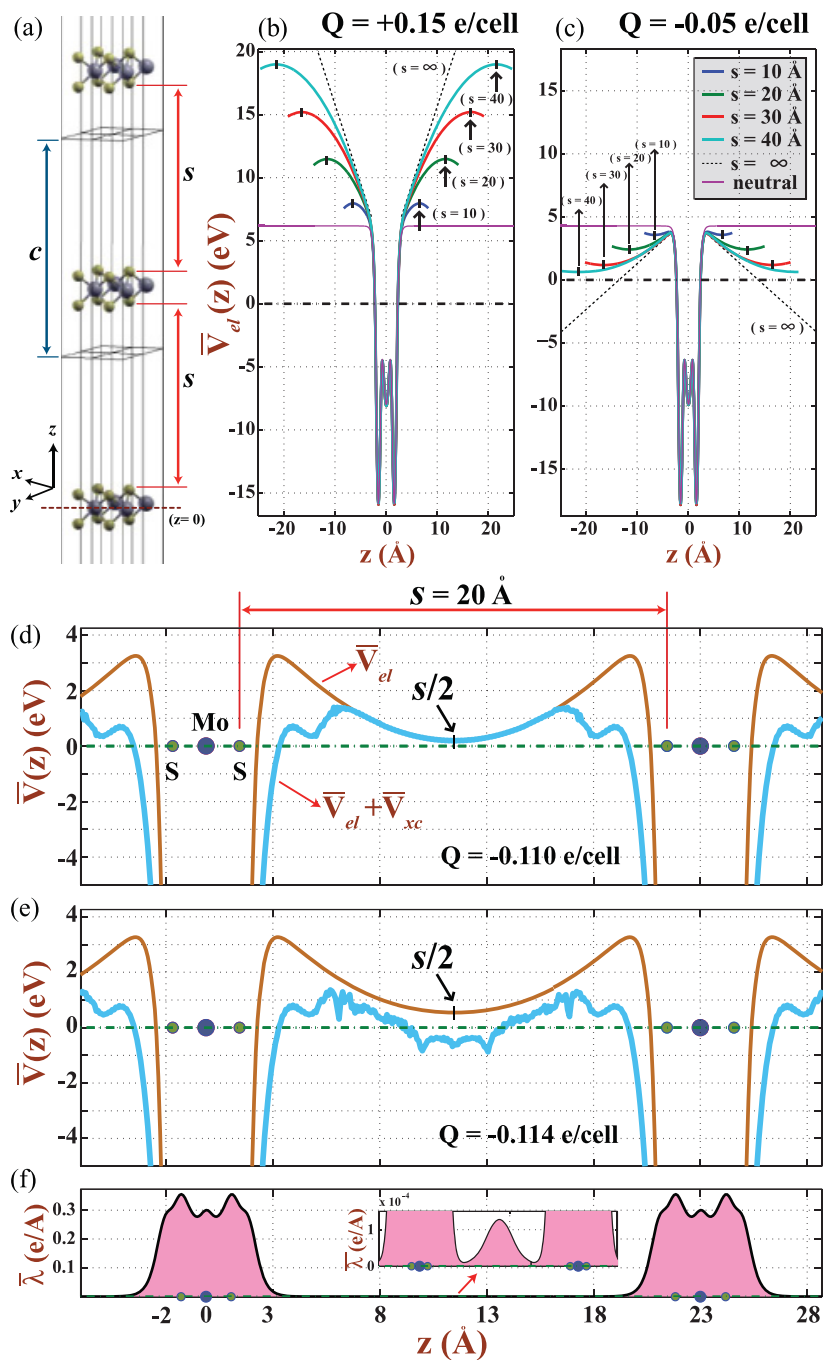

FIG. 1. (Color online) (a) Description of supercell geometry used to treat 2D single layer $\mathrm{MoS}_{2} . c$ and $s$ are the supercell lattice constant and vacuum spacing between adjacent layers, respectively. The $z$ axis is perpendicular to the layers. (b) Self-consistent potential energy of positively charged ( $Q>0$ per cell), periodically repeating $\mathrm{MoS}_{2}$ single layers, which is planarly averaged along the $z$ direction. $\bar{V}_{e l}(z)$ is calculated using different vacuum spacings $s$, as specified in the inset. The planarly averaged potential energy of a single and infinite $\mathrm{MoS}_{2}$ layer is schematically shown by linear dashed lines in the vacuum region. The zero of energy is set at the Fermi level, which is indicated by dash-dotted lines. (c) $\bar{V}_{e l}(z)$ of negatively charged ( $Q<0$ per cell) and periodically repeating $\mathrm{MoS}_{2}$ single layers. The averaged potential energy of an infinite $\mathrm{MoS}_{2}$ single layer is shown by the linear and dashed lines in the vacuum region. (d) Variation of $\bar{V}_{e l}(z)$ and total potential energy including electronic and exchangecorrelation potential, $\bar{V}_{e l}(z)+\bar{V}_{x c}(z)$, between two negatively charged $\mathrm{MoS}_{2}$ layers corresponding to $Q=-0.110 \mathrm{e} / \mathrm{unit}$ cell before the spilling of electrons into vacuum. The spacing between $\mathrm{MoS}_{2}$ layers is $s=20 \AA$. (e) Same as (d) but $Q=-0.114 \mathrm{e} /$ unit cell, where the total potential energy dips below $E_{F}$ and hence excess electrons start to fill the states localized in the potential well between two $\mathrm{MoS}_{2}$ layers. (f) Corresponding planarly averaged charge density $\bar{\lambda}$. Accumulation of the charge at the center of $s$ is resolved in a fine scale. Arrows indicate the extremum points of $\bar{V}_{e l}(z)$ in the vacuum region for $Q>0$ and $Q<0$ cases. 
plane-wave basis set and the repeating layers separated by the vacuum space $s$. Despite that, the method may provide a reasonable description of the negatively charged layers until the minimum of the well dips below the Fermi level. According to this picture, the escaping of electrons out of the material is delayed in relatively short $s$. On the other hand, the interaction between layers prevents one from using an excessively short $s$. Earlier, this limitation of the method was usually overlooked. The critical value of negative charge depends on $s$. It should be noted that, for $s=20 \AA$, electrons start to escape from the graphene layer for $Q=-4.03 \times 10^{13} \mathrm{e} / \mathrm{cm}^{2}$, even though larger doping of $4 \times 10^{14} \mathrm{e} / \mathrm{cm}^{2}$ has been attained for graphene on an $\mathrm{SiO}_{2}$ substrate. ${ }^{38}$

In the case of positive charging, even if $\bar{V}_{e l}(z)$ is not linear and does not increase to $+\infty$, the periodic boundary conditions using sufficiently large $s$ can provide a realistic description of a charged systems, since the wave functions in the vacuum region rapidly decay under a high and wide potential barrier. Therefore, the calculated wave functions and electronic energies are not affected even if $\bar{V}_{e l}(z)$ is smaller than the electronic potential corresponding to infinite vacuum spacing. We demonstrate our point of view by directly solving the Schrödinger equation to obtain the wave functions and energy eigenvalues for the planarly averaged 1D potentials of single layer and three-layer graphene corresponding to $s=12.5,25$, and $50 \AA$ in Fig. 2 . One sees that the large difference, $\Delta \bar{V}_{e l}(z)=\bar{V}_{e l, s=50 \AA}(z)-\bar{V}_{e l, s=12.5 \AA}(z)$ do not affect the occupied states at their tail region in the vacuum spacing; the energy difference is only $5 \mathrm{meV}$ (which cannot be resolved from the figure) between the smallest and largest vacuum spacing $s$, which is smaller than the accuracy limit of DFT calculations. As one expects, the dependence on the vacuum spacing increases for excited states, which have relatively larger extension and hence are affected by $\Delta \bar{V}_{e l}(z)$.

By taking the above limitations of the method in negative charging into account, we now examine the effect of charging of single layers of graphene, $\mathrm{CF}, \mathrm{CH}, \mathrm{BN}$, and $\mathrm{MoS}_{2}$ on their electronic structure and bond lengths. In Fig. 3 the changes in band structure with charging are significant within DFT. For example, the band gap (i.e., the energy gap between the top of the valence band and the minimum of the conduction band) of neutral single-layer BN increases from 4.61 to 5.12 and to $5.54 \mathrm{eV}$ as $Q$ increases from $Q=0$ to $+0.2 \mathrm{e} /$ cell and to $+0.4 \mathrm{e} /$ cell, respectively. The increase of the band gap occurs due to the fact that the electronic potential energy becomes deeper with increasing electron depletion. For $Q>$ 0 , the Fermi level dips in the valance band and creates holes.

In contrast, parabolic free-electron-like bands, which occur above the vacuum level in the neutral case, start to descend as a result of negative charging $(Q<0)$ and eventually they touch the Fermi level. Upon increasing $Q$ these parabolic bands start to be occupied and hence part of $Q$ is transferred to the quantum well in the vacuum region. This way the rate of accumulation of excess charge in the conduction band of a single-layer nanostructure recedes. Even if these parabolic bands appear to touch the Fermi level in the same band structure in the $\left(k_{x}, k_{y}\right)$ plane they are physically separated from the states of the single-layer honeycomb structure under study. As was mentioned before, this situation is an artifact (a) 1-layer $\mathrm{BN}$

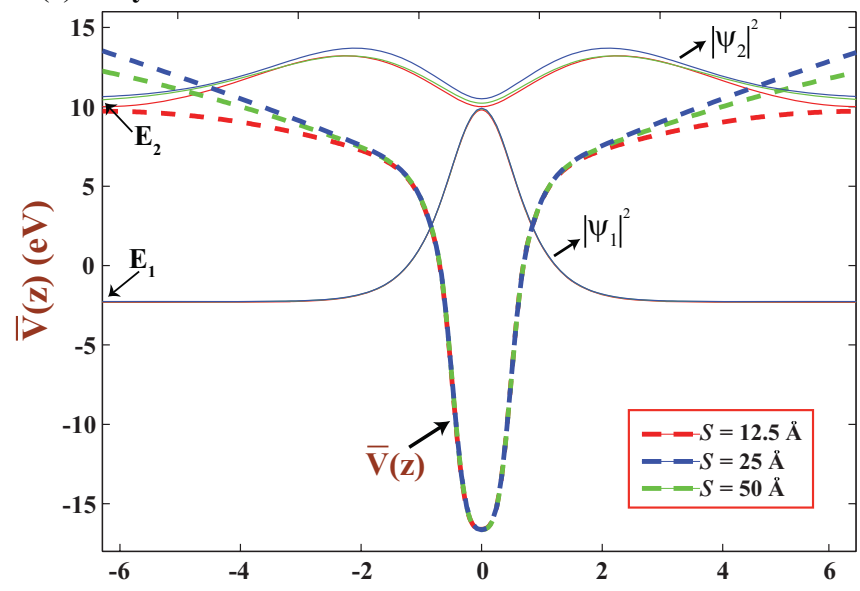

(b) 3-layer BN

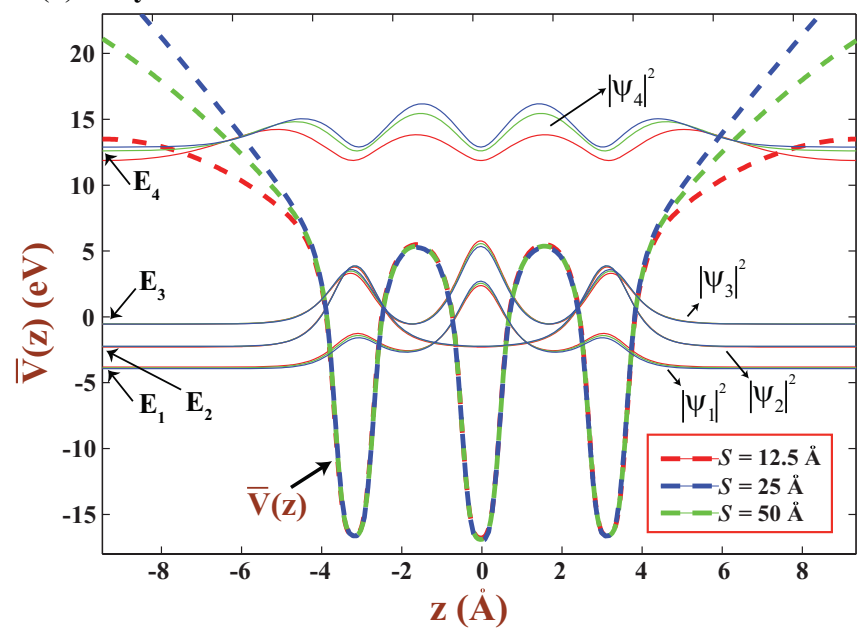

FIG. 2. (Color online) (a) Energy eigenvalues of the occupied electronic states, $E_{i}$ and corresponding $\left|\Psi_{i}(z)\right|^{2}$ are obtained by the numerical solution of the Schrödinger equation of a planarly averaged, 1D electronic potential energy of single-layer graphene for $s=12.5 \AA$, $25 \AA$, and $50 \AA$ shown by dashed lines. (b) Same as (a) for three-layer graphene. Zeros of $\left|\Psi_{i}(z)\right|^{2}$ at large $z$ are aligned with the corresponding energy eigenvalues.

of periodic boundary conditions and can be viewed as the vanishing of the work function. We note that, in the case of a negatively charged, finite-size single layer, the excess electrons are hindered from spilling out to the vacuum by a wide tunneling barrier, even if $\bar{V}_{e l}(z)$ is lowered below the Fermi level in vacuum for large $z$.

Incidentally, for both $Q>0$ and $Q<0$, the spin-polarized calculations carried out for single layers of graphene, $\mathrm{CH}, \mathrm{CF}$, $\mathrm{BN}$, and $\mathrm{MoS}_{2}$ did not yield any magnetic state as a result of charging.

Another crucial effect of charging appears in the variation of the bond lengths with $Q$. As shown in Fig. 4(a) the bond length or lattice constants of single-layer graphene, $\mathrm{BN}, \mathrm{CH}, \mathrm{CF}$, and $\mathrm{MoS}_{2}$ increase with increasing positive charge density $\bar{\sigma}$. The elongation of the bond length is slow for small $\bar{\sigma}$, but increases quickly when $\bar{\sigma} \gtrsim 1 \mathrm{C} / \mathrm{m}^{2}$. The bonds get longer when the electrons are removed from the system and hence bonds 

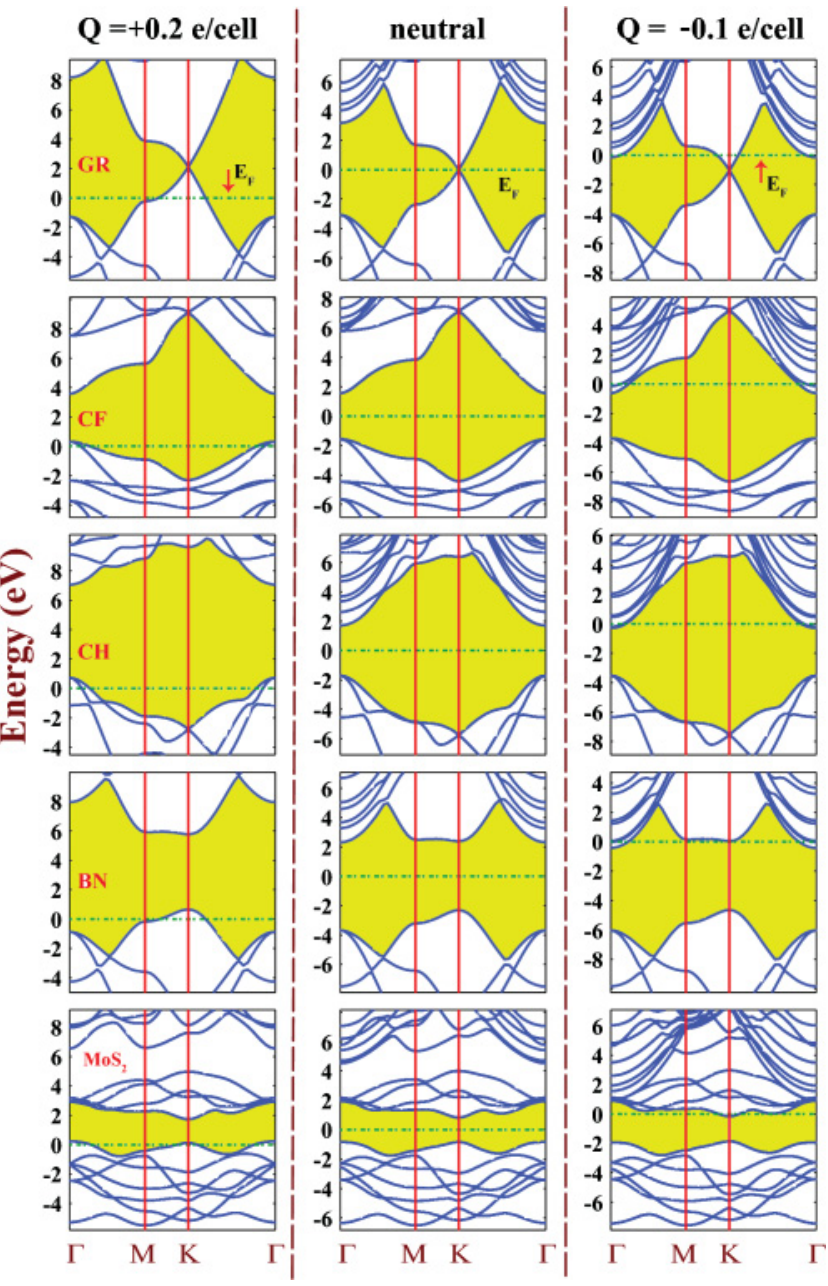

FIG. 3. (Color online) Energy-band structures of a 2D single layer of graphene $\mathrm{C}$, fluorographene $\mathrm{CF}$, graphane $\mathrm{CH}, \mathrm{BN}$, and $\mathrm{MoS}_{2}$ calculated for $Q=+0.2 \mathrm{e} /$ cell, $Q=0$ (neutral), and $Q=$ $-0.10 \mathrm{e} / \mathrm{cell}$. The zero of energy is set at the Fermi level, which is indicated by dash-dotted lines. The band gap is shaded. Note that band gap increases under positive charging. Parabolic bands descending and touching the Fermi level for $Q<0$ are free-electron-like bands. Band calculations are carried out for $s=20 \AA$.

become weaker. The contour plots of total charge density in a plane passing through $\mathrm{C}-\mathrm{C}$ and $\mathrm{B}-\mathrm{N}$ bonds of graphene and $\mathrm{BN}$ honeycomb structures in Figs. 4(b) and 4(c) show that the charge density between atoms becomes weaker with increasing electron depletion. Weakening of bonds can have crucial consequences as phonon softening and is observable through the Raman spectrum. In fact, the Raman active mode of graphene calculated using density functional perturbation theory is at $1524 \mathrm{~cm}^{-1}$ and shifts down to $1510 \mathrm{~cm}^{-1}$ for $Q=$ $+0.2 \mathrm{e} /$ cell, and to $1311 \mathrm{~cm}^{-1}$ for $Q=0.4 \mathrm{e} /$ cell. To confirm whether the elongation of bonds dominates the Raman shift of graphene, we calculated the Raman active modes of neutral graphene having the same lattice constant of graphene when charged by $Q=+0.4 \mathrm{e} /$ cell. In this case the Raman active mode shifted to $1274 \mathrm{~cm}^{-1}$, which is close to the Raman active mode calculated for graphene charged with $Q=+0.4 \mathrm{e} / \mathrm{cell}$. We also note that the excessive charging of single-layer materials considered in this paper leads to instability. This (a)



(b) Graphene

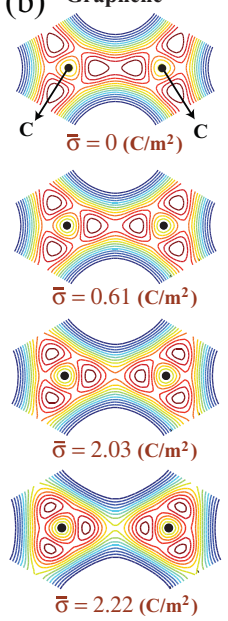

(c) BN

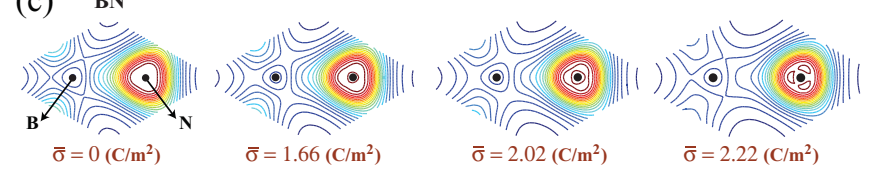

FIG. 4. (Color online) (a) Variation of the ratio of lattice constants $a$ of positively charged single-layer graphene, $\mathrm{BN}, \mathrm{CH}, \mathrm{CF}$, and $\mathrm{MoS}_{2}$ to their neutral values $a_{o}$ with the average surface charge density, $\bar{\sigma}$. The unit cell and the lattice vectors are described by inset. (b) The charge density contour plots in a plane passing through a $\mathrm{C}-\mathrm{C}$ bond. (c) Same as B-N bond.

is revealed from phonon dispersion calculations. For example, neutral graphene, which normally has a stable planar structure and positive frequencies of acoustical branches in BZ, starts to attain imaginary frequencies of long-wavelength acoustical modes at excessive charging. Weakening of the graphene layer is expected to be reflected in its elastic properties, in particular in its stiffness. ${ }^{39}$

\section{EXFOLIATION OF LAYERED BN AND MOS}

We next investigate the exfoliation of single layer BN and $\mathrm{MoS}_{2}$ from their layered bulk crystal through charging. We model a three-layer slab (sheet) of $\mathrm{BN}$ and $\mathrm{MoS}_{2}$ as part of their layered bulk crystal. We considered only three-layer slabs in order to cut the computation time, since the model works also for thicker slabs consisting of 6-10 layers of graphene. ${ }^{15}$ Energy minimizations of neutral sheets relative to stacking geometry are achieved. Stacking of three-layer $\mathrm{BN}$ and $\mathrm{MoS}_{2}$ slabs complies with the stacking of layers in three-dimensional (3D) layered $\mathrm{BN}^{7}$ and $\mathrm{MoS}_{2}$ crystals. ${ }^{9}$ In these slabs, the layers are held together mainly by attractive $\mathrm{vdW}$ interactions of a few hundreds of meV and any repulsive interaction overcoming it leads to exfoliation. When electrons are injected to or removed from the slab, the Fermi level shifts up or down and cross the conduction or valance band of the insulator and attribute to it a metallic character. At the end, the excess charge by itself accumulates on the surfaces of the metallic slab inducing a repulsive Coulomb interaction between the outermost layers of the slab. Here we consider positive charging only, since in the case of negative charging the excess charges quickly spill into the vacuum before the exfoliation sets in. 

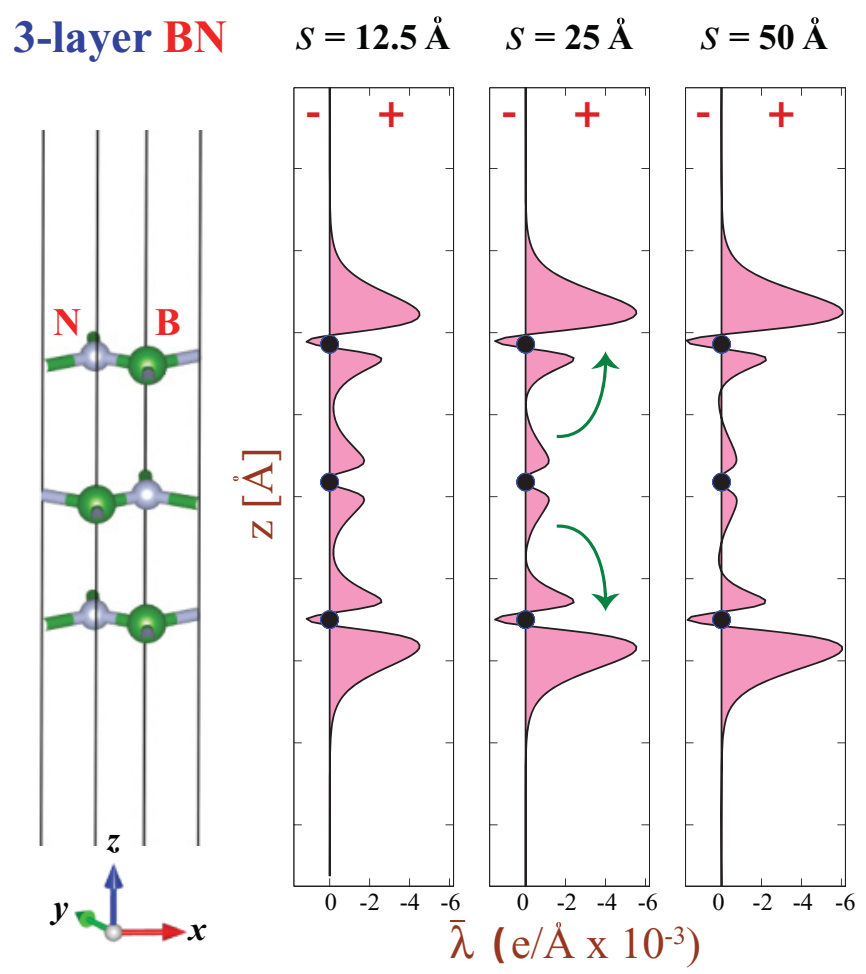

FIG. 5. (Color online) Variation of planarly averaged positive excess charge $\bar{\lambda}(z)$ along $z$ axis perpendicular to the BN layers calculated for different $s$. As $s$ increases more excess charge is transferred from the center region to the surface planes.

The amount of charge in the unit cell, which is necessary for the onset of exfoliation, is defined as the threshold charge $Q_{e}$. Threshold charges are calculated for three-layer slabs of graphene, BN, and $\mathrm{MoS}_{2}$ for $s=20 \AA$ and $s=50 \AA$. Results presented in Table I indicate that the amount of threshold charge decreases with increasing $s$. This confirms our arguments in Sec. III that in positive charging large vacuum space, $s$, is favored. The mechanism underlying this finding is summarized in Fig. 5 where we show the linear charge density $\bar{\lambda}(z)$ calculated for different $s$ values of a three-layer BN. For small $s$, the excess charge accumulates mainly at surfaces of the slab, also with some significant fraction inside the slab. However, as $s$ increases some part of $Q$ is transferred from inside to the outer surface, giving rise to the increase of the charge accumulation at the surface. At the end, for the same level of charging, the induced Coulomb repulsion increases with increasing $s$. Accordingly, the same slab requires a relatively smaller amount of threshold charge $Q_{e}$ to be exfoliated, if $s$ is taken large.

In Fig. 6 we present the variation of the cohesive energy of the three-layer $\mathrm{BN}$ slab relative to three free $\mathrm{BN}$ layers for the neutral $Q=0$ and positive charged $Q>0$ cases as a function of distance $L$ between the outermost BN atomic planes of the three-layer $\mathrm{BN}$ slab. The cohesive energy for a given $L$ is obtained from the following expression: $E_{C}=E_{T}$ (three-layer $\left.\mathrm{BN}\right)-3 E_{T}$ (single-layer $\left.\mathrm{BN}\right)$. The total energy of the single-layer $\mathrm{BN}, E_{T}$ (single layer) is calculated in a smaller supercell to keep the density of the background charge the same. The cohesive energy of the neutral slab in
TABLE I. Dependence of the threshold charges on the vacuum spacing $s(\AA)$ between three-layer slabs. Threshold charge $Q_{e}$ (e/cell) where exfoliation sets in and corresponding threshold average surface charge density $\bar{\sigma}_{e}=Q_{e} / A\left(\mathrm{C} / \mathrm{m}^{2}\right)$ are calculated for positive charged three-layer graphene, $\mathrm{BN}$, and $\mathrm{MoS}_{2}$ sheets for $s=50 \AA$ and $s=$ $20 \AA$. The numbers of valence electrons per unit cell of the slab are also given in the second column.

\begin{tabular}{lccc}
\hline \hline System & No. of e & $Q_{e}(\mathrm{e} /$ cell $)$ & $\bar{\sigma}\left(\mathrm{C} / \mathrm{m}^{2}\right)$ \\
\hline Three-layer Graphene $(s=50)$ & 24 & +0.160 & +0.49 \\
Three-layer Graphene $(s=20)$ & 24 & +0.205 & +0.62 \\
Three-layer BN $(s=50)$ & 24 & +0.225 & +0.66 \\
Three-layer BN $(s=20)$ & 24 & +0.320 & +0.94 \\
Three-layer $\mathrm{MoS}_{2}(s=50)$ & 54 & +0.322 & +0.57 \\
Three-layer $\mathrm{MoS}_{2}(s=20)$ & 54 & +0.480 & +0.86 \\
\hline \hline
\end{tabular}

equilibrium is $\sim 302 \mathrm{meV} /$ cell. If the spacings of layers (i.e., $L)$ starts to increase, an attractive force $F_{\perp}=-\partial E_{T} / \partial L$ acts to restore the equilibrium spacing. $F_{\perp}(L)$ first increases with increasing $L$, passes through a maximum, and then decays to zero. In Fig. 6 we also show how the minimum of cohesive energy decreases and moves to relatively large spacings with increasing $Q$. Concomitantly, the maximum of the attractive force for a given $Q, F_{\perp, \max }$ decreases with increasing $Q$ and eventually becomes zero. This give rise to the exfoliation. We note that despite the limitations set by the neutralizing uniform charge on the total energy, the cohesive energies calculated for different charge levels reveal useful qualitative information on the effects of charging.

In Fig. 7(a) we show isosurfaces of excess positive charge densities of three-layer $\mathrm{BN}$ and $\mathrm{MoS}_{2}$ slabs. These slabs become metallic upon extracting electrons (i.e., upon positive charging) and excess charges reside at both surfaces of slabs. As shown in Fig. 7(b), the total energy rises with increasing charging or average charge density $\bar{\sigma}$. In compliance with Fig. 6, the separation $L$ between surface layers increases. The sharp drop of $\Delta E$ at $Q_{e}$ or $\bar{\sigma}_{e}$ indicates the onset of exfoliation due to the repulsive Coulomb force pulling them to exfoliate. In Fig. 7(c), $L$ increases with increasing charging as discussed for Fig. 6. The increments of $L$ exhibits a stepwise behavior for BN. This is also artifact of the method, where forces are calculated within preset threshold values.

The variation of $L$ of $\mathrm{MoS}_{2}$ slab with $Q>0$ displays a different behavior due to charge transfer from Mo to $\mathrm{S}$ atoms. The exfoliation due to the static charging can be explained by a simple electrostatic model, where the outermost layers of slabs is modeled by uniformly charged planes, which yields a repulsive interaction independent of their separation distance, [i.e., $F \propto Q^{2} /\left(A \epsilon_{0}\right)$ ], where $\epsilon_{0}$ is the static dielectric constant. ${ }^{15}$ Calculated forces differ from the classical force due to the screening effects of excess charge residing inside the slabs.

\section{DISCUSSIONS AND CONCLUSIONS}

In this study, the threshold values of static charge $Q_{e}$ to be implemented in slabs to achieve exfoliation are quite high. Such high static charging of layers can be achieved locally through the tip of a scanning tunnelling microscope 

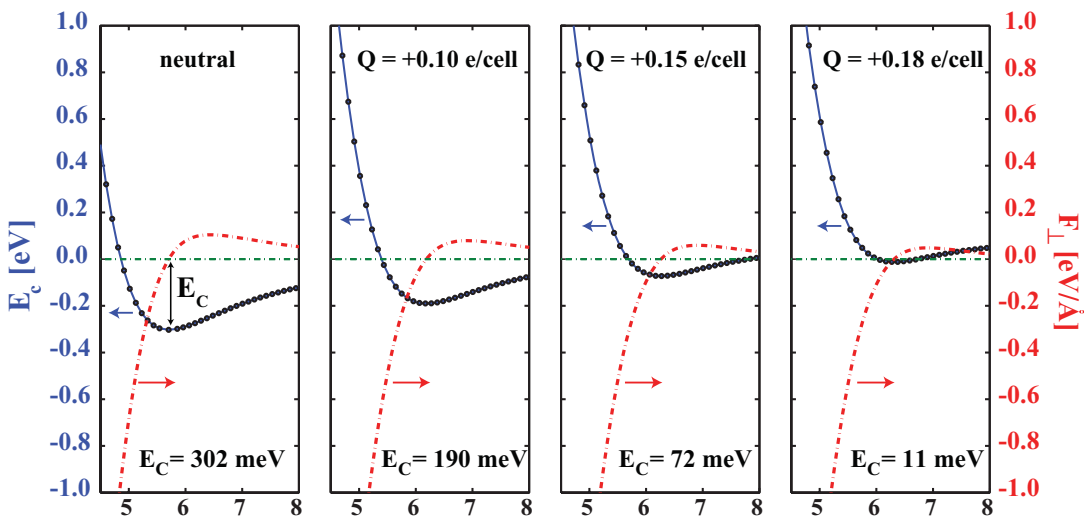

FIG. 6. (Color online) Variation of cohesive energy and perpendicular force $F_{\perp}$ in three-layer BN slab as a function of the distance $L$ between the surfaces. Energies and forces are calculated for different levels of excess positive charge $Q$ (e/unit cell). Zero of energy corresponds to the energy as $L \rightarrow \infty$. or electrolytic gate. ${ }^{38}$ The dissipation of locally created excess charge in materials may involve a decay time $\tau_{D}$. Relatively longer $\tau_{D}$ can induce a local instability and the desorption of atoms from nanoparticles. Experimentally, ultrafast graphene ablation was directly observed by means of electron crystallography. ${ }^{16}$ Carriers excited by ultrashort laser pulse transfer energy to strongly coupled optical phonons. Graphite undergoes a contraction, which is subsequently followed by an expansion leading eventually to laser-driven ablation. ${ }^{16}$ More recently, the understanding of photoexfoliation has been proposed, where exposure to femtosecond laser pulses has led to athermal exfoliation of intact graphenes. ${ }^{17}$ Based on timedependent DFT calculations (TD-DFT), it is proposed that the femtosecond laser pulse rapidly generates a hot electron gas at $\sim 20000 \mathrm{~K}$, while graphene layers are vibrationally cold. The hot electrons spill out, leaving behind a positively charged graphite slab. The charge deficiency accumulated at the top and bottom surfaces leads to athermal excitation. ${ }^{17}$ The exfoliation in static charging described in Fig. 7 is in compliance with the understanding of photoexcitation revealed from previous TD-DFT calculations, ${ }^{17}$ since the driving force which leads to the separation of graphenes from graphite is mainly related with electrostatic effects in both methods.
In summary, the present study investigated the effects of charging on the structural and electronic properties of singlelayer graphene, graphene derivatives, $\mathrm{BN}$, and $\mathrm{MoS}_{2}$, which have a honeycomb structure. We conclude that, while caution has to be exercised in studies involving negative charging using large vacuum spacing, positive charging can be treated safely using large vacuum spacing.

We found that, upon positive charging, the band gaps of single layers of $\mathrm{BN}$ and $\mathrm{MoS}_{2}$ increase and the unit cells are enlarged. Consequently, the phonons become softer. The charging of $\mathrm{BN}$ and $\mathrm{MoS}_{2}$ slabs were also studied. While these slabs are wide-band semiconductors, they become metallic upon positive charging. Consequently, excess charges are accumulated on the surfaces of slabs and induce a repulsive force between the outermost layers. With increasing positive charging the spacing between these layers increases, which eventually ends with exfoliation.

\section{ACKNOWLEDGMENTS}

We thank S. Cahangirov for helpful discussions. We acknowledge partial financial support from The Academy of Science of Turkey (TUBA) and TUBITAK
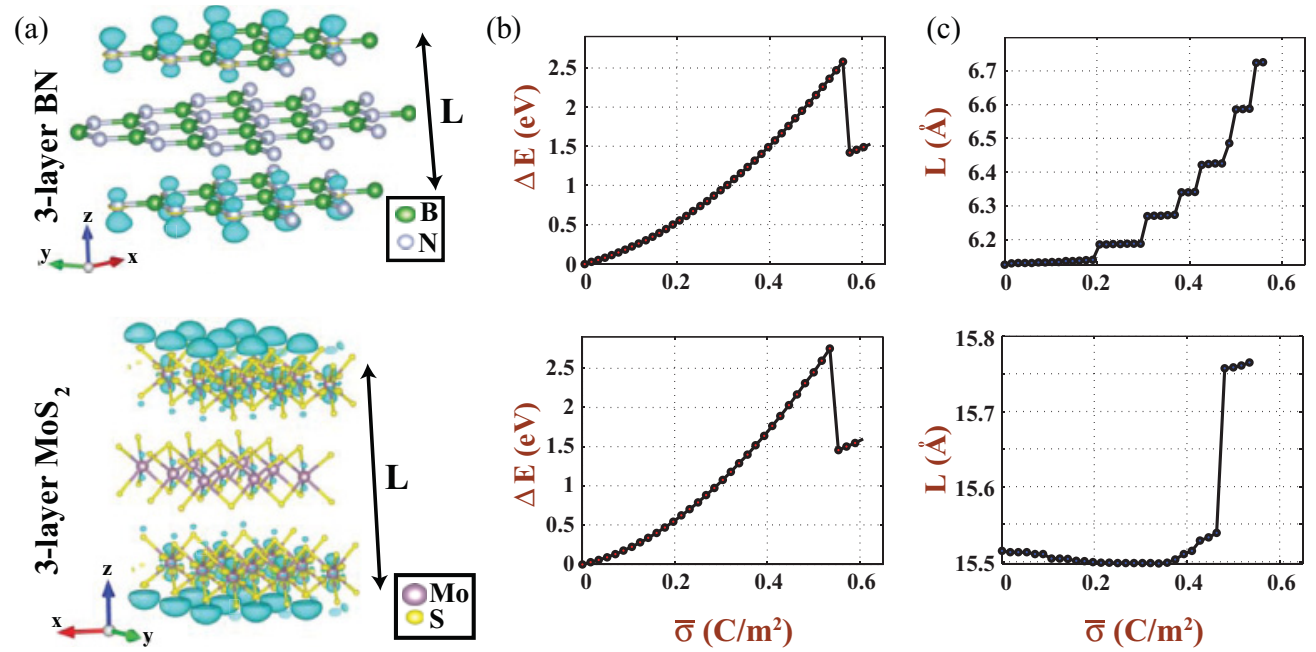

FIG. 7. (Color online) Exfoliation of outermost layers from layered $\mathrm{BN}$ and $\mathrm{MoS}_{2}$ slabs by positive charging of slabs. (a) Turquoise isosurfaces of excess positive charge density. (b) Change in total energy with excess surface charge density. (c) Variation of $L$ of slabs with charging. 
through Grant No. 108234. Part of the computational resources has been provided by TUBITAK ULAKBIM,
High Performance and Grid Computing Center (TR-Grid e-Infrastructure). *ciraci@fen.bilkent.edu.tr

${ }^{1}$ K. S. Novoselov, A. K. Geim, S. V. Morozov, D. Jiang, Y. Zhang, S. V. Dubonos, I. V. Grigorieva, and A. A. Firsov, Science 306, 666 (2004).

${ }^{2}$ D. C. Elias, R. R. Nair, T. M. G. Mohiuddin, S. V. Morozov, P. Blake, M. P. Halsall, A. C. Ferrari, D. W. Boukhvalov, M. I. Katsnelson, A. K. Geim, and K. S. Novoselov, Science 323, 610 (2009).

${ }^{3}$ H. Sahin, C. Ataca, and S. Ciraci, Appl. Phys. Lett. 95, 222510 (2009).

${ }^{4}$ R. R. Nair, W. Ren, R. Jalil, I. Riaz, V. G. Kravets, L. Britnell, P. Blake, F. Schedin, A. S. Mayorov, S. Yuan, M. I. Katsnelson, H.-M. Cheng, W. Strupinski, L. G. Bulusheva, A. V. Okotrub, I. V. Grigorieva, A. N. Grigorenko, K. S. Novoselov, and A. K. Geim, Small 6, 2877 (2010).

${ }^{5}$ H. Sahin, M. Topsakal, and S. Ciraci, Phys. Rev. B 83, 115432 (2011).

${ }^{6}$ C. Jin, F. Lin, K. Suenaga, and S. Iijima, Phys. Rev. Lett. 102, 195505 (2009).

${ }^{7}$ M. Topsakal, E. Akturk, and S. Ciraci, Phys. Rev. B 79, 115442 (2009). A comprehensive list of current references on layered and single-layer $\mathrm{BN}$ is given in this paper.

${ }^{8}$ K. F. Mak, C. Lee, J. Hone, J. Shan, and T. F. Heinz, Phys. Rev. Lett. 105, 136805 (2010).

${ }^{9}$ C. Ataca, H. Sahin, E. Akturk, and S. Ciraci, J. Phys. Chem. C 115, 3934 (2011); C. Ataca and S. Ciraci, ibid. 115, 13303 (2011); C. Ataca, M. Topsakal, E. Akturk, and S. Ciraci, ibid. 115, 16354 (2011). A comprehensive list of current references on layered and single layer $\mathrm{MoS}_{2}$ is given in this paper.

${ }^{10}$ K. S. Novoselov, A. K. Geim, S. V. Morozov, D. Jiang, M. I. Katsnelson, I. V. Grigorieva, S. V. Dubonos, and A. A. Firsov, Nature (London) 438, 197 (2005).

${ }^{11}$ X. Wang, X. Li, L. Zhang, Y. Yoon, P. K. Weber, H. Wang, J. Guo, and H. Dai, Science 324, 768 (2009).

${ }^{12}$ T. O. Wehling, K. S. Novoselov, S. V. Morozov, E. E. Vdovin, M. I. Katsnelson, A. K. Geim, and A. I. Lichtenstein, Nano Lett. 8, 173 (2008).

${ }^{13}$ H. Sevincli, M. Topsakal, E. Durgun, and S. Ciraci, Phys. Rev. B 77, 195434 (2008).

${ }^{14}$ C. Ataca, E. Aktürk, S. Ciraci, and H. Ustunel, Appl. Phys. Lett. 93, 043123 (2008).
${ }^{15}$ M. Topsakal and S. Ciraci, Appl. Phys. Lett. 98, 131908 (2011).

${ }^{16}$ F. Carbone, P. Baum, P. Rudolf, and A. H. Zewail, Phys. Rev. Lett. 100, 035501 (2008).

${ }^{17}$ Y. Miyamoto, H. Zhang, and D. Tomanek, Phys. Rev. Lett. 104, 208302 (2010).

${ }^{18}$ O. O. Ekiz, M. Urel, H. Guner, A. K. Mizrak, and A. Dana, ACS Nano 5, 2475 (2011).

${ }^{19}$ K. T. Chan, H. Lee, and M. L. Cohen, Phys. Rev. B 83, 035405 (2011).

${ }^{20}$ K. T. Chan, H. Lee, and M. L. Cohen, Phys. Rev. B 84, 165419 (2011).

${ }^{21}$ A. M. Suarez, L. R. Radovic, E. Bar-Ziv, and J. O. Sofo, Phys. Rev. Lett. 106, 146802 (2011).

${ }^{22}$ P. E. Blochl, Phys. Rev. B 50, 17953 (1994).

${ }^{23}$ J. P. Perdew, J. A. Chevary, S. H. Vosko, K. A. Jackson, M. R. Pederson, and D. J. Singh, and C. Fiolhais, Phys. Rev. B 46, 6671 (1992).

${ }^{24}$ S. Grimme, J. Comp. Chem. 27, 1787 (2006).

${ }^{25}$ G. Kresse and J. Furthmuller, Phys. Rev. B 54, 11169 (1996).

${ }^{26}$ M. Leslie and N. J. Gilan, J. Phys. C 18, 973 (1985).

${ }^{27}$ G. Makov and M. C. Payne, Phys. Rev. B 51, 4014 (1995).

${ }^{28}$ I. Dabo, B. Kozinsky, N. E. Singh-Miller, and N. Marzari, Phys. Rev. B 77, 115139 (2008).

${ }^{29}$ J. Neugebauer and M. Scheffler, Phys. Rev. B 46, 16067 (1992).

${ }^{30}$ P. Gava, M. Lazzeri, A. M. Saitta, and F. Mauri, Phys. Rev. B 79, 165431 (2009).

${ }^{31}$ C. L. Fu and K. M. Ho, Phys. Rev. Lett. 63, 1617 (1989).

${ }^{32}$ P. E. Blochl, J. Chem. Phys. 103, 7422 (1995).

${ }^{33}$ P. A. Schultz, Phys. Rev. B 60, 1551 (1999); Phys. Rev. Lett. 84, 1942 (2000).

${ }^{34}$ S. Heinze, X. Nie, S. Bigel, and M. Weinert, Chem. Phys. lett. 315, 167 (1999).

${ }^{35}$ A. Y. Lozovoi and A. Alavi, Phys. Rev. B 68, 245416 (2003).

${ }^{36}$ J. S. Filhol and M. Neurock, Angew. Chem. 118, 416 (2006); C. D. Taylor, S. A. Wasileski, J.-S. Filhol, and M. Neurock, Phys. Rev. B 73, 165402 (2006).

${ }^{37}$ S. Schnur and A. Gross, Catal. Today 165, 129 (2011).

${ }^{38}$ D. K. Efetov and P. Kim, Phys. Rev. Lett. 105, 256805 (2010).

${ }^{39}$ M. Topsakal, S. Cahangirov, and S. Ciraci, Appl. Phys. Lett. 96, 091912 (2010). 